\title{
Effects of Nonlinear Micromagnetic Coupling on a Weak-Field Magnetoimpedance Sensor
}

\author{
Kwaku Eason and Kok-Meng Lee, Fellow, IEEE \\ G. W. Woodruff School of Mechanical Engineering, Georgia Institute of Technology, Atlanta, GA 30332-0405
}

\begin{abstract}
We present a general harmonic formulation that takes into account, explicitly, the effects of micromagnetics for modeling the magnetic fields in a magnetoimpedance (MI) sensor element. We first relax assumptions commonly made to derive closed form solutions from a decoupled set of linear equations. We then solve numerically (using a meshless method formulated in point-collocation) the Maxwell and the Landau-Lifshitz-Gilbert equations simultaneously for the real and imaginary parts of the magnetic fields and magnetization in the context of a cylindrical amorphous MI sensor element. Comparing our results of the effects of coupling the equations of motion against published experimental data, we found striking differences both quantitatively and qualitatively between the coupled nonlinear and decoupled linear models. The coupled nonlinear harmonic formulation presented here results in improved accuracy and more consistent qualitative behavior in accordance to reported experimental observations, particularly in the weak field regime. Also presented here are spin wave amplitude distributions showing spatial dispersion within MI elements structures, which represents information lost in decoupled formulations.
\end{abstract}

Index Terms-Magnetization, magnetoimpedance, meshless methods, MI sensor, micromagnetic.

\section{INTRODUCTION}

H IGHLY sensitive sensors capable of detecting very weak magnetic fields have a spectrum of applications ranging from small memory devices to biomedical applications. Among these are sensors based on the magnetoimpedance (MI) effect which is a sensitive realignment of a periodic magnetization in response to an external magnetic field. Devices like MI-effect sensors that have experimentally demonstrated an absolute resolution on the order of $10^{-10}$ Tesla $(\mathrm{T})$ or better [1] offer the potential of high sensitivity at low cost. The complex behavior of MI effects ultimately suggests the design of high-sensitivity MI sensors requires a good understanding of both electrodynamics and micromagnetic effects on the magneto impedance.

Interest in MI has generated a flurry of research since the discovery of very large MI (GMI) effects in amorphous ferromagnetic wires [2]-[4] and thin films [5] with small magnetic fields at relatively low frequencies in the early 1990s. More recently, MI effects have been studied in multilayer films and composites [6], [7]. More than 100 promising applications of GMI have been suggested in [8]. Despite its potential, MI sensor designs have relied heavily on experimental techniques. Early experimental results have been described qualitatively by way of the impedance model derived by Landau and Lifshitz [9] for magnetic wires, which has revealed a dependence on a relation between the transverse magnetic permeability and measured external field. The manner of finding this relationship has been the distinction between many previous models. One method is based on the calculation of ac complex impedance with a rigid domain structure in both wires and films [10], [11], and for a flexible wall model [12]. Other methods for deriving a lumped-parameter-based effective permeability were based on an energy approach, for example, [13] and [14].

Digital Object Identifier 10.1109/TMAG.2008.922780

Color versions of one or more of the figures in this paper are available online at http://ieeexplore.ieee.org.
The interest to extend MI sensors for detecting very weak magnetic signals in biomedical applications, along with the challenging requirements of greater speed and areal density in magnetic storage elements, has considerably increased the effort of researchers in the investigation of magnetization dynamics. Many of the analyses are based on the dynamic model proposed by Landau and Lifshitz in 1935, and successively modified by Gilbert in 1955 . Guided by the solution to a linearized harmonic form of the Landau-Lifshitz-Gilbert (LLG) equation, a permeability model was presented in [15] for the case of axial (and transverse) anisotropy in a cylindrical element. The solutions [15], [16] provide a permeability tensor, from which the effective transverse permeability scalar is computed. The permeability so derived depends on the case of assumed anisotropy direction. In [17], a similar model for the case of transverse anisotropy in wires was presented. Additional assumptions (such as small amplitudes in the ac source) are commonly made in order to reduce the micromagnetic dynamics to a linear form. Most of these existing approaches have essentially utilized the decoupled impedance model (Landau-Lifshitz impedance for wires) derived from the decoupled Maxwell equations along with a permeability tensor (or effective scalar) for modeling the MI effect. While the linearized LLG equation is more tractable, the lumped-parameter permeability which generally yields only first-order accuracy is inadequate to characterize the MI effect in response to very weak external fields. For this reason, we have developed a general formulation (that relaxes key assumptions leading to linearization) for characterizing the magnetic fields in MI sensors.

The remainder of this paper offers the following.

1) We offer a general harmonic formulation (that takes into account, explicitly, the effects of micromagnetics) for modeling the magnetic fields in an MI sensor element. While relaxing assumptions commonly made in the literature, this coupled nonlinear model provides a means to explicitly solve the Maxwell and LLG equations simultaneously for the real and imaginary parts of the magnetic field and magnetization. 
2) We present a model deploying a meshless method (MLM) formulation using point collocation [18] with the HP-cloud shape functions [19] for solving the coupled nonlinear model. We validate the numerical method (referred to here as HP-PC MLM) by comparing results against those using a Galerkin finite-element method (FEM), which shows excellent agreement and an advantage of HP-PC MLM in terms of computational time.

3) We investigate the effects of micromagnetic coupling on the MI effect by comparing the coupled nonlinear model computed using the HP-PC MLM against the decoupled linear model, which has a closed form solution. The computed impedance voltage, which has been based on a circular amorphous wire for the case of axial anisotropy, is compared against published experimental data [20]. As will be shown, unlike the decoupled linear model that is invalid in the weak external field regime, the nonlinear coupled model agrees very well qualitatively with the experimental data. In addition, the analytical models discussed offer an intuitive means to visualize the effect of micromagnetics particularly in the weak field regime.

\section{EQUATIONS OF MOTION}

The main characteristics of the MI effects in amorphous wires and ribbons can be partly understood in the frame of classical electrodynamics:

$$
\frac{\partial \mathbf{B}}{\partial t}=-\frac{1}{\sigma}(\nabla \times \nabla \times \mathbf{H}) \text { where } \mathbf{B}=\mu_{0} \mathbf{H}+\mathbf{M}
$$

where $\mathbf{B}$ and $\mathbf{H}$ are the magnetic flux density and field intensity, respectively; $\mathbf{M}$ is the magnetization; $\mu_{o}$ is the permeability of free space; and $\sigma$ is the bulk electrical conductivity.

The magnetization "motion" is governed by the Landau-Lifshitz and Gilbert equations originally proposed by Landau and Lifshitz, and successively modified by Gilbert [16]:

$$
\frac{\partial \mathbf{M}}{\partial t}=-\gamma \mathbf{M} \times \mathbf{H}_{T}+\frac{\alpha}{M_{s}} \mathbf{M} \times \frac{\partial \mathbf{M}}{\partial t}
$$

where $\gamma$ is the gyromagnetic ratio; $\alpha$ is the phenomenological Gilbert damping constant; and $M_{\mathrm{S}}$ is the saturation magnetization. In (2), $\mathbf{H}_{T}$ is the total effective field vector:

$$
\mathbf{H}_{T}=\mathbf{H}+\mathbf{H}_{K}+\mathbf{H}_{X}+\mathbf{H}_{D}
$$

where $\mathbf{H}$ is the sum of the applied external fields; and $\mathbf{H}_{K}, \mathbf{H}_{\mathrm{X}}$, and $\mathbf{H}_{\mathrm{D}}$ are the effective fields taking into account the effects of anisotropy, exchange, and demagnetization, respectively. The effective anisotropy field $\mathbf{H}_{K}$ (that is generally a function of temperature) is given by [21]:

$$
\mathbf{H}_{K}=\left(H_{K} / M_{s}\right)\left(\mathbf{M} \cdot \mathbf{e}_{\mathbf{k}}\right) \mathbf{e}_{\mathbf{k}}
$$

where $H_{K}=\left|\mathbf{H}_{K}\right|$; and $\mathbf{e}_{k}$ is the unit vector in the direction of the material preferred anisotropy. The exchange field $\mathbf{H}_{\mathrm{X}}$ is given by

$$
\mathbf{H}_{X}=A_{X} \nabla^{2} \mathbf{M}
$$

where $A_{\mathrm{X}}$ is the exchange stiffness. The demagnetization field $\mathbf{H}_{\mathrm{D}}$ is characterized by the Poisson equation

$$
\mathbf{H}_{\mathrm{D}}=-\nabla \varphi_{D} \quad \text { and } \quad \mu_{0} \nabla^{2} \varphi_{D}=\nabla \cdot \mathbf{M} .
$$

Since $\mathbf{M} \times(\partial \mathbf{M} / \partial t)=\operatorname{Skew}(\mathbf{M})(\partial \mathbf{M} / \partial t)$, (2) can be written as

$$
[\boldsymbol{\Gamma}] \frac{\partial \mathbf{M}}{\partial \mathrm{t}}=-\gamma \mathbf{M} \times \mathbf{H}_{T}
$$

where

$$
\begin{aligned}
{[\mathbf{\Gamma}] } & =[\mathbf{I}]-\frac{\alpha}{M_{s}} \operatorname{Skew}(\mathbf{M}) \\
\operatorname{Skew}(\mathbf{M}) & =\left[\begin{array}{ccc}
0 & -M_{3} & M_{2} \\
M_{3} & 0 & -M_{1} \\
-M_{2} & M_{1} & 0
\end{array}\right]
\end{aligned}
$$

and $[\mathbf{I}]$ is the identity matrix. Equations (1) and (7), equivalent to the Maxwell-Landau-Lifshitz-Gilbert (MLLG) equations [22], are referred to here as the Maxwell skewed-LLG (MsLLG) equations. The MsLLG equations are a coupled set of nonlinear PDEs that describe the $\mathbf{H}$ and $\mathbf{M}$ fields within the MI element.

Once the $\mathbf{H}$ and $\mathbf{M}$ fields are found, the inductive voltage $\mathrm{V}_{\mathrm{L}}$ across the MI element is given by Faraday's law of electromagnetic induction. For a stationary circuit in a time varying magnetic field

$$
V_{L}=-\frac{d \Phi}{d t} \quad \text { where } \Phi=\int_{S}\left(\mu_{0} \mathbf{H}+\mathbf{M}\right) \cdot d \mathbf{s}
$$

where $S$ is the magnetic flux crossing surface area.

\section{A. Coupled Nonlinear Harmonic Formulation}

For the problem of the MI element under a dc external field, $\mathbf{M}$ and $\mathbf{H}$ are assumed to take the form

$$
\begin{aligned}
\mathbf{H} & =\mathbf{H}_{e}+\mathbf{H}_{\sim} e^{-j \omega t+\phi}=\mathbf{H}_{e}+\mathbf{H}_{R}+j \mathbf{H}_{I} \\
\mathbf{M} & =\mathbf{M}_{d}+\mathbf{M}_{\sim} e^{-j \omega t+\phi_{M}}=\mathbf{M}_{d}+\mathbf{M}_{R}+j \mathbf{M}_{I}
\end{aligned}
$$

where $\phi$ and $\phi_{M}$ are phase angles with respect to the ac source. The field $\mathbf{H}_{e}$ is a dc field, representing that which is to be measured, while $\mathbf{M}_{d}$ is given by the magnetization equilibrium state. The components $\mathbf{H}_{\sim}$ and $\mathbf{M}_{\sim}$ are both ac components of their respective vector fields, at frequency $\omega$. Similarly

$$
\begin{aligned}
& {[\boldsymbol{\Gamma}]=\left[\boldsymbol{\Gamma}_{R}\right]+j\left[\boldsymbol{\Gamma}_{I}\right]} \\
& \varphi_{D}=\varphi_{D R}+j \varphi_{D I} .
\end{aligned}
$$

Substitution of (9a, b) and (10a, b) into (1), (7) and (6) leads to the following partial differential equations (PDEs) given in (11)-(13) in vector form:

$$
\begin{aligned}
& \nabla \times \nabla \times \mathbf{H}_{R}+\sigma \omega\left(\mu_{0} \mathbf{H}_{I}+\mathbf{M}_{I}\right)=0 \\
& \nabla \times \nabla \times \mathbf{H}_{I}-\sigma \omega\left(\mu_{0} \mathbf{H}_{R}+\mathbf{M}_{R}\right)=0 \\
& \frac{\omega}{\gamma}\left(\left[\boldsymbol{\Gamma}_{I}\right] \mathbf{M}_{R}+\left[\boldsymbol{\Gamma}_{R}\right] \mathbf{M}_{I}\right) \\
& \quad=\mathbf{M}_{I} \times \mathbf{H}_{T I}-\left(\mathbf{M}_{d}+\mathbf{M}_{R}\right) \times \mathbf{H}_{T R}
\end{aligned}
$$




$$
\begin{aligned}
& \frac{\omega}{\gamma}\left(\left[\boldsymbol{\Gamma}_{R}\right] \mathbf{M}_{R}-\left[\boldsymbol{\Gamma}_{I}\right] \mathbf{M}_{I}\right) \\
& \quad=\mathbf{M}_{I} \times \mathbf{H}_{T R}+\left(\mathbf{M}_{d}+\mathbf{M}_{R}\right) \times \mathbf{H}_{T I} \\
& \mu_{0} \nabla^{2} \varphi_{D R}=\nabla \cdot \mathbf{M}_{R} \\
& \mu_{0} \nabla^{2} \varphi_{D I}=\nabla \cdot \mathbf{M}_{I} .
\end{aligned}
$$

Equations (11)-(13), along with (3)-(5), are a coupled nonlinear model, which explicitly solves the Maxwell and LLG equations simultaneously for the real and imaginary parts of the magnetic field $\left(\mathbf{H}_{R}\right.$ and $\left.\mathbf{H}_{I}\right)$, magnetization $\left(\mathbf{M}_{R}\right.$ and $\left.\mathbf{M}_{I}\right)$, and demagnetization field potential $\left(\varphi_{D R}\right.$ and $\left.\varphi_{D I}\right)$.

\section{B. Decoupled Linear Formulation}

A common approach to reduce the coupled nonlinear equations, (1) and (7), to a more tractable form is to decouple the electromagnetic from the magnetization motion equations by approximating $\mathbf{M} \approx[\chi] \mathbf{H}$, where $[\chi]$ is the magnetic susceptibility tensor and hence

$$
\mathbf{B} \approx[\boldsymbol{\mu}] \mathbf{H} \quad \text { where }[\boldsymbol{\mu}]=\mu_{0}[\mathbf{I}]+[\chi] .
$$

With (14), the Maxwell (1) no longer explicitly depends on $\mathbf{M}$; and the linear system can be solved for an impedance model in terms of the permeability tensor $[\boldsymbol{\mu}]$, which can be derived independently from the LLG equation as a function of the external field.

A permeability model $[\boldsymbol{\mu}]$ (in terms of the applied/external magnetic field $\mathbf{H}$ ) can be derived in closed form from the linearized LLG equation for a quasi-static problem with an axial anisotropy, which satisfies the following commonly made assumptions.

1) The amplitude of the $a c$ field is small compared to that of the dc fields.

2) $\mathbf{H}_{T d}$ and $\mathbf{M}_{d}$ are known a priori at the equilibrium state:

$$
\mathbf{M}_{d} \times \mathbf{H}_{T d}=0
$$

where $\mathbf{H}_{T d}$ is the dc component of $\mathbf{H}_{T}$.

3) Exchange and demagnetization effects can be neglected:

$$
\mathbf{H}_{T} \approx \mathbf{H}+\mathbf{H}_{K} .
$$

Under assumptions 1 and 2, the linearized LLG equation can be obtained by removing all products of $a c$ terms:

$$
\left[\boldsymbol{\Gamma}_{R}\right]\left(\partial \mathbf{M}_{\sim} / \partial t\right)=-\gamma\left(\mathbf{M}_{d} \times \mathbf{H}_{T \sim}+\mathbf{M}_{\sim} \times \mathbf{H}_{T d}\right) .
$$

Substituting (9b) into (4), the anisotropy field is written as

$$
\mathbf{H}_{K}=\left[\mathbf{M}_{K}\right]\left(\mathbf{M}_{d}+\mathbf{M}_{\sim}\right) .
$$

The matrix, $\left[\mathbf{M}_{\mathrm{K}}\right]$ is given in (20). It follows from (9a) with assumption 3 that

$$
\begin{aligned}
\mathbf{H}_{T d} & =\mathbf{H}_{e}+\left[\mathbf{M}_{K}\right] \mathbf{M}_{d} \\
\mathbf{H}_{T \sim} & =\mathbf{H}_{\sim}+\left[\mathbf{M}_{K}\right] \mathbf{M}_{\sim} .
\end{aligned}
$$

With 18(a, b), (16) can be solved analytically for the magnetic susceptibility. The permeability tensor $[\boldsymbol{\mu}]$ can be calculated from (14b).

Permeability Tensor for the Case of Axial Anisotropy: For the case of axial anisotropy, the equilibrium magnetization is given by

$$
\mathbf{M}_{d}=\left[\begin{array}{lll}
0 & 0 & M_{s}
\end{array}\right]^{T}
$$

and

$$
\left[\mathbf{M}_{K}\right]=\left[\begin{array}{ccc}
0 & 0 & 0 \\
0 & 0 & 0 \\
0 & 0 & H_{K}
\end{array}\right] .
$$

Equations (19) and (20) imply Skew $\left(\mathbf{M}_{d}\right)\left[\mathbf{M}_{K}\right]=0$. Upon solving (16) for $[\chi],[\boldsymbol{\mu}]$ is given by (14b) as

$$
\frac{[\boldsymbol{\mu}]}{\mu_{0}}=[\mathbf{I}]+\frac{\gamma}{\mu_{0}}\left[-j \omega\left[\boldsymbol{\Gamma}_{d}\right]+\gamma\left[\mathrm{S}_{2}\right]\right]^{-1}\left[\mathrm{~S}_{1}\right]
$$

where $\left[\boldsymbol{\Gamma}_{d}\right]=[\mathbf{I}]-\left(\alpha / M_{s}\right)$ Skew $\left(\mathbf{M}_{d}\right) ;\left[S_{1}\right]=\operatorname{Skew}\left(\mathbf{M}_{d}\right)$; and $\left[S_{2}\right]=$ Skew $\left(\mathbf{H}_{e}+\left[\mathbf{M}_{K}\right] \mathbf{M}_{d}\right)$. The permeability tensor takes the form [16]

$$
[\boldsymbol{\mu}]=\left[\begin{array}{ccc}
\mu & j \mu_{a} & 0 \\
-j \mu_{a} & \mu & 0 \\
0 & 0 & \mu_{0}
\end{array}\right]
$$

where $\mu$ and $\mu_{\mathrm{a}}$ depend on the external magnetic field. In SI units,

$$
\mu_{a}=\mu_{o}+\frac{\omega_{H} \gamma M_{s}}{\omega_{H}^{2}-\omega^{2}} \quad \text { and } \quad \mu=\frac{\omega \gamma M_{s}}{\omega_{H}^{2}-\omega^{2}}
$$

where $\omega_{H}=\gamma\left(H_{e}+H_{k}\right)-j \omega \alpha M_{s}$.

Impedance Model for a Cylindrical Magnetic Wire: Derived from the Maxwell (1), the impedance was given by Landau and Lifshitz [9] for a circular magnetic wire (Fig. 1)

$$
Z\left(\omega, \mu_{\theta}\right)=\frac{1}{2} k r_{o} R_{\mathrm{dc}} \frac{J_{0}\left(k r_{o}\right)}{J_{1}\left(k r_{o}\right)}
$$

and

$$
k=\sqrt{j \sigma_{c} \mu_{\theta} \omega}=(1+j) / \delta_{s}
$$

where $r_{\mathrm{o}}$ is the outer radius of the MI element, $R_{\mathrm{dc}}$ is the dc resistance, $J_{0}$ and $J_{1}$ are Bessel functions of the first kind, $\mu_{\theta}$ is the transverse magnetic permeability; and $\delta_{\mathrm{s}}$ is the classical skin depth. The impedance model (24) explicitly depends on the lumped-parameter transverse permeability $\mu_{\theta}$ and the source frequency $\omega$. The circular component of $[\boldsymbol{\mu}]$ relating the circular component of $\mathbf{B}$ to such components of $\mathbf{H}$ is given by

$$
\mu_{\theta+}=\mu+\mu_{a} .
$$

Another expression for the lumped-parameter transverse permeability used in [15], [23] is given by

$$
\mu_{\theta \perp}=\mu-\mu_{a}^{2} / \mu .
$$




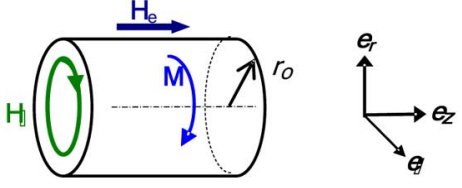

Fig. 1. Cylindrical MI element.

TABLE I

SIMULATION PARAMETERS

\begin{tabular}{|c|c|c|c|c|c|c|}
\hline $\begin{array}{l}M_{\mathrm{s}} \\
(\mathrm{T})\end{array}$ & $\begin{array}{c}\mathrm{I}_{\mathrm{s}} \\
(m A)\end{array}$ & $\begin{array}{c}\sigma \\
(k \Omega-m)^{-1}\end{array}$ & $\begin{array}{c}H_{K} \\
(\mathrm{~A} / \mathrm{m})\end{array}$ & $\alpha$ & $\begin{array}{c}A_{\mathrm{X}} \\
(\mathrm{J} / \mathrm{m})\end{array}$ & $\gamma$ \\
\hline 1.35 & 15 & 67.367 & 0.78 & 0.01 & $10^{-11}$ & 21,020 \\
\hline
\end{tabular}

\section{Numerical Solution of Coupled Nonlinear Model}

The full (coupled and nonlinear) model generally has 14 component variables: $\varphi_{D R}, \varphi_{D I}, H_{R i}, H_{I i}, M_{R i}$, and $M_{I i}$, where $i=1,2,3$ denote the three spatial coordinates. The solutions to the coupled nonlinear model are solved numerically using a meshless method. The procedures for a numerical formulation in point collocation (strong form) and weak form for solving a boundary value problem using a MLM can be found in [18].

Boundary Conditions for a Cylindrical Magnetic Wire: To determine a unique and relevant solution for the MI effect equations of motion, an appropriate set of boundary conditions (BCs) must be specified. Without loss of generality, we consider, as an illustration, a cylindrical amorphous wire shown in Fig. 1, where

$$
\mathbf{H}_{e}=H_{e} \mathbf{e}_{\mathbf{z}}
$$

Since $\mathbf{M}$ and $\mathbf{H}$ only vary radially, the radial components of $(11 \mathrm{a}, \mathrm{b})$ lead to

$$
\mu_{o}\left(H_{R r}+j H_{I r}\right)=-\left(M_{R r}+j M_{I r}\right) .
$$

The remaining 12 coupled nonlinear component equations of (11a, b), (12a, b), and (13a, b) are solved numerically along with the following boundary conditions:

$$
\begin{aligned}
& r=0 \quad \partial \mathbf{H} / \partial r=\partial \mathbf{M} / \partial r=\partial \varphi_{D} / \partial r=0 \\
& r=r_{o} \quad H_{R \theta}=I_{s} / 2 \pi R ; \quad H_{I \theta}=0 \\
& H_{R z}=0 ; \quad H_{I z}=0 \\
& \partial \mathbf{M} / \partial r=0 ; \\
& \mu_{0} \partial\left(\phi_{D R}+j \phi_{D I}\right) / \partial r=M_{R r}+j M_{I r}
\end{aligned}
$$

Equation (31e) assumes the free-spinning condition [24]. Note that the field intensity at the interface $\left(r=r_{o}\right)$ can be obtained by integrating Ampere's law over the cross-sectional area because of the current source, which leads to (31); thus, the far-field condition is not needed.

Computation for Cylindrical Magnetic Wire: Numerical simulation has been performed on a circular amorphous wire (diameter of $30 \mu \mathrm{m}$ and length of $5 \mathrm{~mm}$ ) with an ac source of $15 \mathrm{~mA}$ at $1 \mathrm{MHz}$ over the external field range 0 to $1000 \mathrm{~A} / \mathrm{m}$ so that computed results can be compared against published experimental data [20]. The values for the parameters used in the numerical investigation are summarized in Table I, where $M_{\mathrm{S}}$ is computed from the volume compositional average using published values for cobalt and iron [25].

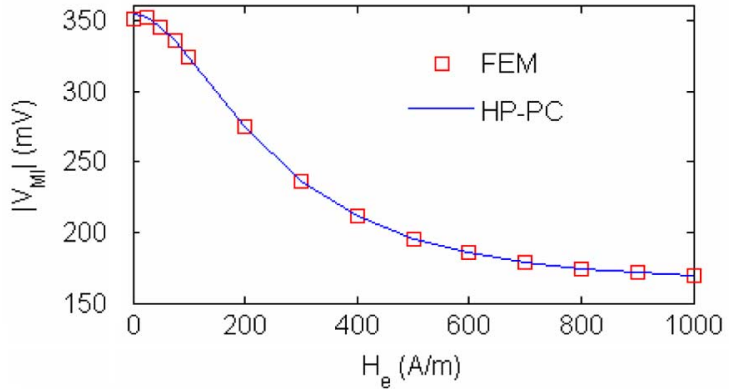

(a)

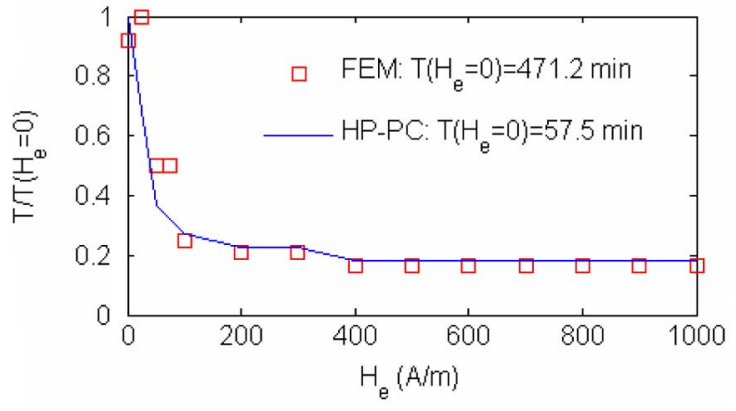

(b)

Fig. 2. Computed $\left|\mathrm{V}_{\mathrm{MI}}\right|$ versus $\mathrm{H}_{\mathrm{e}}, \mathrm{n}=55$ nodes, and computation time $\mathrm{T}$. (a) $\left|\mathrm{V}_{\mathrm{MI}}\right|$ as a function of $H_{e}$. (b) Computation time, T.

Since all computations are performed on a desktop PC, both strong form (or point collocation method) and weak form formulations along with several basis functions were preliminarily tested for computation efficiency leading to the choice of MLM formulated using a point-collocation method [18] with the HP-cloud shape functions [19] referred to here as HP-PC MLM. Computed using a Newton solver with added auto-damping to treat the variable stiffness, the HP-PC MLM was implemented in MATLAB. In each of the computational iterations, the norm of the approximate error $e_{a}$ is calculated as a convergence metric; the computation was terminated when $e_{a}<1 \mathrm{e}-8$.

A key interest in the MI sensor is its response to the external field $H_{e}$ over the region from 0 to near saturation. The sensor response is often measured in terms of the voltage magnitude across the MI sensor, $\left|\mathrm{V}_{\mathrm{MI}}\right|$. Recall $(1 \mathrm{~b})$ provides the magnetic flux density $\mathbf{B}$ in terms of $\mathbf{H}$ and $\mathbf{M}$. The circular component of B determines the inductive voltage (8), while the total voltage is given by

$$
V_{\mathrm{MI}}=\left|I_{s} R_{\mathrm{dc}}+V_{L}\right|
$$

Fig. 2(a) graphs the magnitude of the voltage across the MI element, $\left|\mathrm{V}_{\mathrm{MI}}\right|$, as a function of the external dc field $H_{e}$. It is interesting to note that the computational time to solve the coupled nonlinear model follows the same trend as the MI voltage where the stiffness is increased in the small field region, particularly close the small valued anisotropy assumed to be approximately $0.8 \mathrm{~A} / \mathrm{m}$. To provide a means to validate the numerical computation, the $\left|\mathrm{V}_{\mathrm{MI}}\right|$ results (computed using the HP-PC MLM) are compared with those using FEM (weak form formulation), which show excellent agreement. However, the HP-PC MLM needs significantly less time than the FEM for the same number 
of 55 nodes as compared in Fig. 2(b), where the computation time is normalized to the largest respective time observed at $H_{e}=0$. For the $\left|\mathrm{V}_{\mathrm{MI}}\right|$ at $H_{e}=0$, the FEM took more than $8 \mathrm{~h}$ to compute 55 nodes (or 27 second-order elements) while the HP-PC MLM computed the same result in less than $1 \mathrm{~h}$.

\section{RESULTS AND DISCUSSION}

The effects of coupling between the micromagnetics and electrodynamics on the magnetoimpedance were investigated numerically by comparing the solutions of the coupled nonlinear MsLLG equations against those approximated using the Landau-Lifshitz impedance model (derived from the decoupled Maxwell equation) with the scalar effective permeability computed from the linearized LLG. The study was based on the same wire with values of the parameters summarized in Table I.

\section{A. Results}

The results (computed using the HP-PC MLM) are given in Figs. 3-7:

A) $\left|\mathrm{V}_{\mathrm{MI}}\right|$ is plotted as a function of the external dc field $H_{e}$ in Fig. 3, and as a function of the source frequency $\omega$ in Fig. 4 where $H_{e}=800 \mathrm{~A} / \mathrm{m}$. In Figs. 3 and $4, \mathrm{D}_{1}$ and $\mathrm{D}_{2}$ refer to the solutions of the decoupled linear model with $\mu_{\theta+}$ and $\mu_{\theta \perp}$ given in (26) and (27), respectively. Computed results are compared against experimental data published in [20]; the wire was subjected to a low annealing tension of $2 \mathrm{~kg} / \mathrm{mm}^{2}$.

B) Fig. 5 shows the circular components of both $\mathbf{H}$ (represented as $\mathrm{H}_{\mathrm{t}}$ ) and $\mathbf{B}$ (represented as $\mathrm{B}_{\mathrm{t}}$ ) for $H_{e}=0$ and $900 \mathrm{~A} / \mathrm{m}$ computed from the coupled nonlinear model. The distribution of $\mathbf{B}$ obtained from (11), (12), and (1b) has been computed using an equally distributed nodal set of 55 nodes. With this information, the resulting distributions can be compared in the case of a weak field $\left(H_{\mathrm{e}}=0\right)$ and a near-saturation field $\left(H_{\mathrm{e}}=900 \mathrm{~A} / \mathrm{m}\right)$ so that the assumptions commonly made in deriving the decoupled models can be examined.

C) This physical image leads to a clearer understanding of the qualitative differences between both models. To offer intuitive insights into the very weak field regime, the following results are computed at $H_{e}=0$.

1) Fig. 6(a) and (b) compares the $B_{\theta}(r)$ distribution in the MI element simulated using the coupled and decoupled models. Assuming only radial variation (no circular variation), the transverse component can be presented within the cross-section of the wire to illustrate, in a more physical sense, the distributions of the flux densities within the MI element, where the vector lengths are used to indicate the amplitudes.

2) The relative component contributions to the total effective field $H_{T}$ in (3) are shown in Fig. 7.

\section{B. Observations and Discussions}

Some observations can be made in the comparisons.

- As shown in Fig. 3, the decoupled linear model agrees well with experimental data in predicting $\left|\mathrm{V}_{\mathrm{MI}}\right|$ for relatively large $H_{e}$, but fails to predict the MI sensor response to $H_{e}$

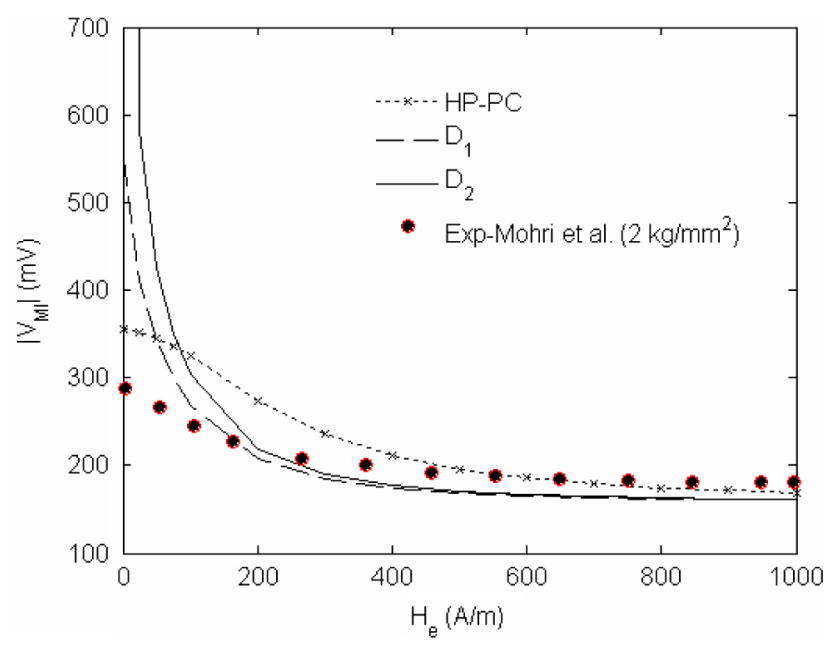

Fig. 3. MI voltages versus external field ( $f=1 \mathrm{MHz})$.

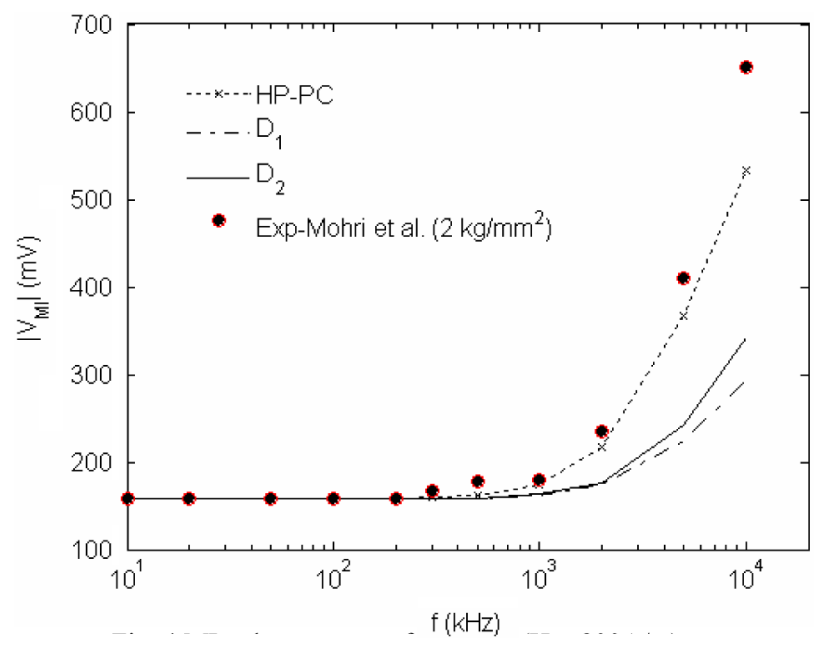

Fig. 4. MI voltages versus frequency $\left(\mathrm{H}_{\mathrm{e}}=800 \mathrm{~A} / \mathrm{m}\right)$.

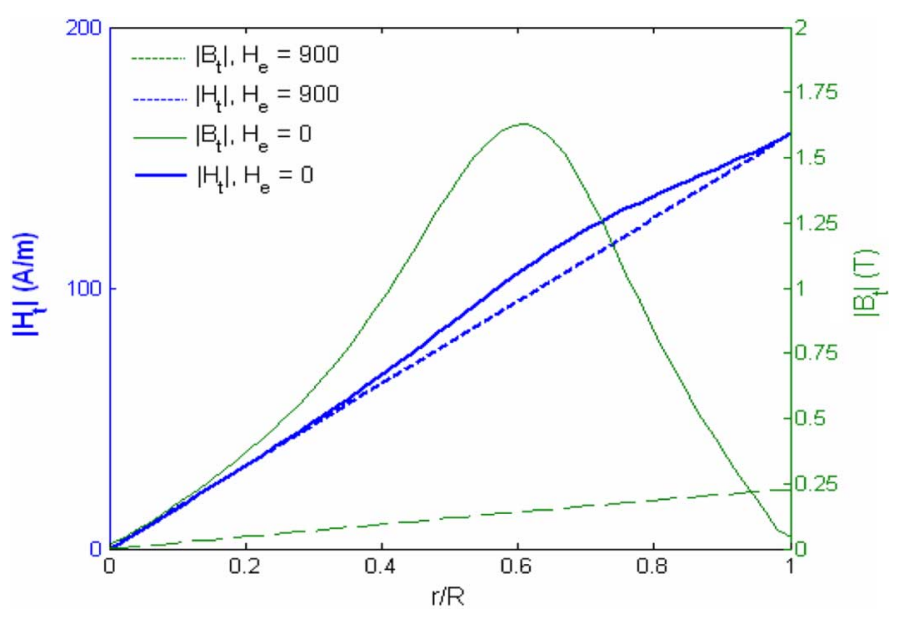

Fig. 5. Effects of $H_{e}$ on the radial variations of $|\mathbf{H}|$ and $|\mathbf{B}|$.

in the weak field region where the harmonic component (and thus the transverse field established by the current) is not smaller than the steady dc external field. This is consistent with assumption 1 for the linearized LLG. Similar discrepancies can be observed in the very high frequency re- 


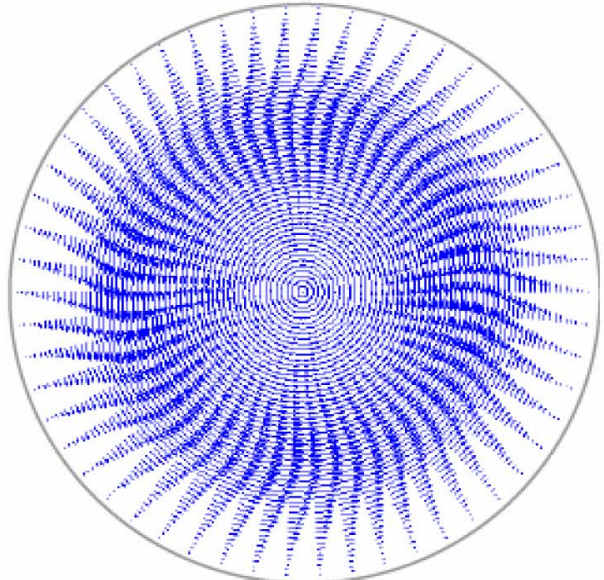

(a)

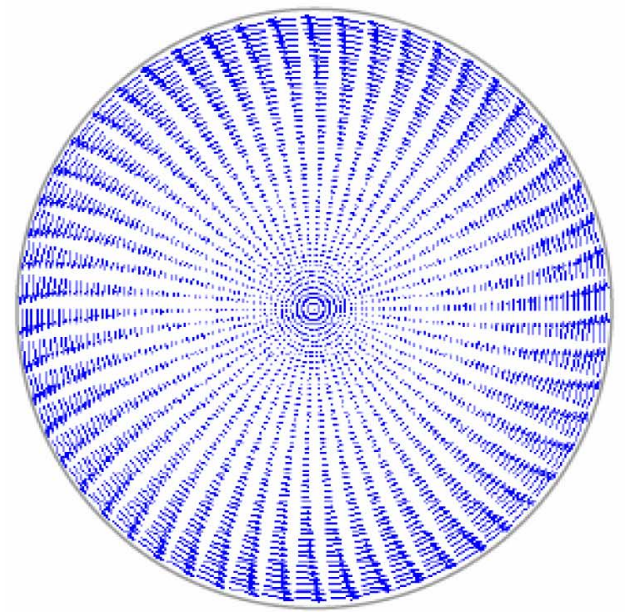

(b)

Fig. 6. Transverse magnetic flux density, $\mathrm{B}_{\theta}$. (a) Coupled nonlinear model, (b) Decoupled linear model.

gion as shown in Fig. 4. Unlike the decoupled linear model that is invalid in the weak field region, the coupled nonlinear model agrees very well qualitatively with the experimental data. The discrepancy near and in the very weak field regime is likely due to domain wall effects that have been neglected in this study.

- Since the decoupled model assumes $\mathbf{M} \approx[\chi] \mathbf{H}$ and $\mathbf{B} \approx$ $\mu_{\theta} \mathbf{H}$, the scalar permeability $\mu_{\theta}$ does not depend on space implying that $\left|B_{\theta}\right|$ and $\left|H_{\theta}\right|$ are parallel in space. Fig. 5 shows that this assumption deteriorates as $H_{e}$ decreases.

- The analytical models offer an intuitive means to visualize the effect of micromagnetics on the transverse magnetic flux density in the weak field regime. Fig. 6(a) and (b) shows two strikingly different $B_{\theta}(r)$ field images predicted by the coupled nonlinear and decoupled linear models, respectively. In Fig. 6, the amplitudes are evidenced from the lengths of the vectors. The nonlinear coupled model [Fig. 6(a)] results in a solution showing the bulk of the transverse induction occurs within the core, which helps explain an experimental observation in Co-based amorphous wires subjected to harmonic fields reported in [26]. Yamasaki et al. [27] observed experimentally in similar

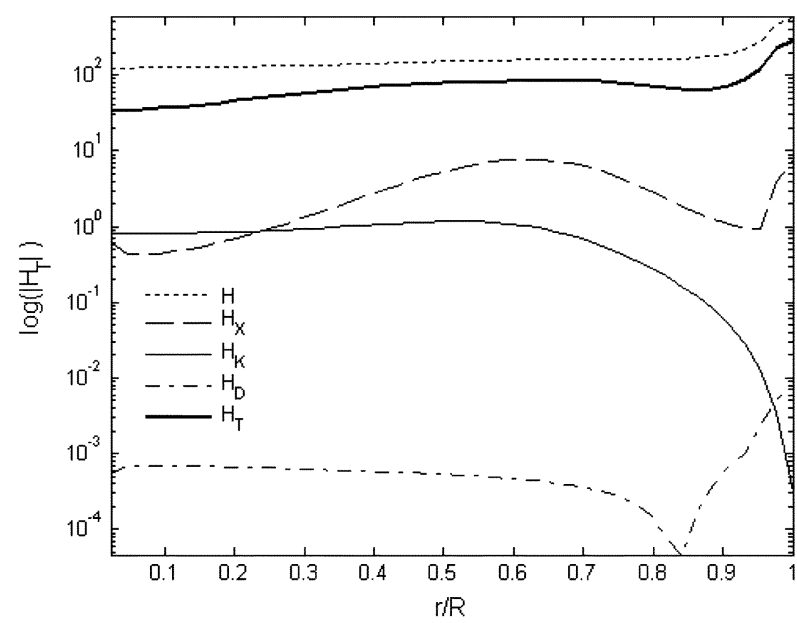

Fig. 7. Relative component contributions to $\mathbf{H}_{\mathrm{T}}\left(\mathrm{H}_{\mathrm{e}}=0\right)$.

wires using Bitter patterns that revealed no switching activity on the wire surface (with observed domain patterns) while induction changes were still observed but the domains participating can only be made visible by polishing away part of the wire. On the other hand, the decoupled linear model [Fig. 6(b)] incorrectly predicts a monotonically increasing induction with maximums near the surface (for the case of weak $H_{e}$ ). One would expect that the transverse magnetic field distribution evolves qualitatively from Fig. 6(a) to (b) over the range $0 \leq H_{e}<1000 \mathrm{~A} / \mathrm{m}$.

- Once the coupled nonlinear equations (11)-(13) are solved, the components that make up the total effective field $\mathbf{H}_{\mathrm{T}}$ can be computed from (4)-(6) for a given external field. Fig. 7 shows (on a log scale) the magnitudes of each component contribution including Zeeman $\mathbf{H}$, anisotropy $\mathbf{H}_{K}$, exchange $\mathbf{H}_{\mathrm{X}}$, and demagnetization $\mathbf{H}_{\mathrm{D}}$ for observing the respective order of magnitudes. As shown in Fig. 7, the magnitude of $\mathbf{H}_{\mathrm{D}}$ is nearly two orders smaller than that of $\mathbf{H}_{K}$ and $\mathbf{H}_{\mathrm{X}}$, in the core of the wire. The dominance of the total effective field by the $\left|\mathbf{H}_{\mathrm{T}}\right|$, evident in Fig. 7, also suggests that the nonlinear dependence of $\mathbf{M}$ on $\mathbf{H}$ can not be neglected; in other words, this dynamic component has a relatively strong contribution and thus, the amplitude of $\mathbf{H}_{\sim}$ is relatively large in its influence on the magnetization. In a decoupled model, the influence of this component is assumed relatively small.

\section{CONCLUSION}

We have presented the effects of coupling both the Maxwell and Landau-Lifshitz-Gilbert equations to model the MI effect using numerical methods. The choice of a point collocation method was shown to offer a considerable advantage in computational efficiency in solving the equations compared to weak formulations. This approach has enabled an investigation that offers intuitive insights into these effects by comparing two analytical (coupled nonlinear and decoupled linear) models against published experimental results [20]. In contrast to a linear approximation that leads to much larger errors in the weak field regime, the coupled nonlinear model agrees very well qualitatively with the experimental data and leads to 
quantitative improvements, also. The discrepancy near the very weak field regime is likely due to domain wall effects that have been neglected in this study. In addition, both models lead to strikingly different distributions within the wire, which provides different qualitative understandings. The role of the skin effect in the weak regime, particularly in a $30 \mu \mathrm{m}$ diameter wire, is a unique artifact of the decoupled linear approximation, while the coupled nonlinear model reveals relatively more intense "activity" within the core, which is somewhat contrary to the phenomena of skin effect. Such a different qualitative feature is consistent with previous (experimental) findings [27], as well. This investigation suggests that the role of coupling is essential for understanding the behaviors of MI devices in the measurement of very weak magnetic fields.

\section{ACKNOWLEDGMENT}

This work was supported in part by an ONR Fellowship.

\section{REFERENCES}

[1] K. Mohri, T. Uchiyama, and L. V. Panina, "Recent advances of micro magnetic sensors and sensing application," Sens. Actuators, vol. 59, pp. 1-8, 1997.

[2] K. Mohri, K. Kawashima, T. Kohzawa, and H. Yoshida, "Magnetoinductive element," IEEE Trans. Magn., vol. 29, no. 2, pp. 1245-1248, Mar. 1993.

[3] R. S. Beach and A. E. Berkowitz, "Giant magnetic field dependent impedance of amorphous FeCoSiB wire," Appl. Phys. Lett., vol. 64, pp. 3652-3654, 1994.

[4] L. V. Panina and K. Mohri, "Magneto-impedance effect in amorphous wires," Appl. Phys. Lett., vol. 65, no. 9, pp. 1189-1191, Aug. 29, 1994.

[5] F. L. A. Machado, B. L. da Silva, S. M. Rezende, and C. S. Martins, "Giant ac magnetoresistance in the soft ferromagnet $\mathrm{Co}_{70.4} \mathrm{Fe}_{4.6} \mathrm{Si}_{15} \mathrm{~B}_{10}$," Phys. Rev. B, vol. 51, pp. 3926-3929, 1995.

[6] P. Ruuskanen, A. Deribas, A. Shtertser, and T. Korkala, "Magnetic properties of $\mathrm{Fe}_{73.5} \mathrm{Cu}_{1} \mathrm{Nb}_{3} \mathrm{Si}_{13.5} \mathrm{~B}_{9}$ alloy compacted by a shock-wave technique," J. Magn. Magn. Mater., vol. 182, pp. 185-192, 1998.

[7] G. V. Kurlyandskaya, J. M. Barandiaran, J. L. Munoz, J. Gutierrez, M. Vazquez, D. Garcia, and V. O. Vas'kovskiy, "Frequency dependence of giant magnetoimpedance effect in $\mathrm{CuBe} / \mathrm{CoFeNi}$ plated wire with different types of magnetic anisotropy," J. Appl. Phys., vol. 87, p. 4822, 2000.

[8] K. Mohri, T. Uchiyama, L. P. Shen, C. M. Cai, and L. V. Panina, "Sensitive micro magnetic sensor family utilizing magneto-impedance (MI) and stress-impedance (SI) effects for intelligent measurements and controls," Sens. Actuators (Phys.), vol. A91, pp. 85-90, 2001.

[9] L. D. Landau, E. M. Lifshitz, and L. P. Pitaevskii, Electrodynamics of Continuous Media, 2nd ed. New York: Academic, 1984, vol. 8.
[10] L. V. Panina, K. Mohri, K. Bushida, and R. Noda, "Giant magneto-impedance and magneto-inductive effects in amorphous alloys," J. Appl. Phys., vol. 76, pp. 6198-6203, 1994.

[11] K. Mohri, L. V. Panina, T. Uchiyama, K. Bushida, and M. Noda, "Sensitive and quick response micro magnetic sensor utilizing magnetoimpedance in Co-rich amorphous wires," IEEE Trans. Magn., vol. 31, no. 2, pp. 1266-1275, Mar. 1995.

[12] P. J. Vulfovich and L. V. Panina, "Magneto-impedance in Co-based amorphous wires and circular domain dynamics," Sens. Actuators (Phys.), vol. A81, pp. 111-116, 2000.

[13] F. L. A. Machado and S. M. Rezende, "A theoretical model for the giant magnetoimpedance in ribbons of amorphous soft-ferromagnetic alloys," J. Appl. Phys., vol. 79, no. 8, pp. 6558-6560, Apr. 15, 1996.

[14] S. S. Yoon, C. G. Kim, H. C. Kim, K. J. Jang, and K. S. Ryu, "Analysis of giant magneto-impedance in terms of anisotropy field in annealed amorphous Co66Fe4NiB14Si15 ribbons," J. Magn. Magn. Mater., vol. 203, pp. 301-303, 1999.

[15] N. A. Usov, A. S. Antonov, and A. N. Lagar'kov, "Theory of giant magneto-impedance effect in amorphous wires with different types of magnetic anisotropy," J. Magn. Magn. Mater., vol. 185, pp. 159-173, 1998.

[16] A. G. Gurevich and G. A. Melkov, Magnetization Oscillations and Waves. Boca Raton, FL: CRC, 1996.

[17] D. Menard, M. Britel, P. Ciureanu, and A. Yelon, "Giant magnetoimpedance in a cylindrical magnetic conductor," J. Appl. Phys., vol. 84, no. 5, pp. 2805-2814, Sep. 1, 1998.

[18] K.-M. Lee, Q. Li, and H. Sun, "Effects of numerical formulation on magnetic field computation using meshless methods," IEEE Trans. Magn., vol. 42, no. 9, pp. 2164-2171, Sep. 2006.

[19] C. A. M. Duarte and J. T. Oden, "HP clouds-a meshless method to solve boundary-value problem," University of Texas, Austin, Tech. Rep. 95-05, 1995.

[20] K. Mohri, K. Bushida, M. Noda, H. Yoshida, L. V. Panina, and T. Uchiyama, "Magneto-impedance element," IEEE Trans. Magn., vol. 31, no. 4, pp. 2455-2460, Jul. 1995.

[21] B. Yang and D. R. Fredkin, "Dynamical micromagnetics by the finite element method," IEEE Trans. Magn., vol. 34, no. 6, pp. 3842-3852, Nov. 1998.

[22] A. Prohl, Computational Micromagnetism. Germany: Teubner, 2001.

[23] N. A. Usov, A. S. Antonov, and A. B. Granovsky, "Theory of giant magneto-impedance effect in composite amorphous wire," J. Magn. Magn. Mater., vol. 171, pp. 64-68, 1997.

[24] G. T. Rado and J. R. Weertman, "Spin-wave resonance in a ferromagnetic metal," J. Phys. Chem. Solids, vol. 11, no. 3-4, pp. 315-333, Oct. 1959.

[25] J. S. Stohr and H. C. , Magnetism: From Fundamentals to Nanoscale Dynamics. New York: Springer, 2006.

[26] A. Hubert and R. Schafer, Magnetic Domains: The Analysis of Magnetic Microstructures. New York: Springer, 1998.

[27] J. Yamasaki, F. B. Humphrey, K. Mohri, H. Kawamura, H. Takamure, and R. Malmhall, "Large Barkhausen discontinuities in Co-based amorphous wires with negative magnetostriction," J. Appl. Phys., vol. 63, no. 8, pp. 3949-3951, Apr. 15, 1988.

Manuscript received December 6, 2007; revised April 2, 2008. Corresponding author: K.-M. Lee (e-mail: kokmeng.lee@ me.gatech.edu). 